\title{
The Use of Serum MMP-9 and TIMP-1 in Acute Coronary Syndrome
}

\author{
Laura Lahdentausta $^{1,2}$ (D) Timo Sorsa ${ }^{1,2,3} \cdot$ Erkki Pesonen $^{4,5} \cdot$ Pirkko Pussinen $^{1,2}$ \\ Received: 31 August 2018 / Accepted: 7 September 2018 / Published online: 24 September 2018 \\ (C) Springer Science+Business Media, LLC, part of Springer Nature 2018
}

Systemically measured matrix metalloproteinase (MMP)-9 and its endogenous inhibitor TIMP-1 are important proteins and inflammatory mediators in acute coronary syndrome (ACS) [4]. Santana and Tanus-Santos [5] argued that the sample material may raise technical issues. They refer to earlier publications in which higher MMP-9 and TIMP-1 concentrations are obtained in serum compared to plasma [2]. Within different plasma samples, MMP-9 and TIMP-1 concentrations are higher when EDTA is used as anticoagulant compared to heparin [3].

In clinical and research use, consistent sample material and processing methods should be used to avoid misinterpretations. It is crucial to use the same sample material and the same processing methods throughout the whole study to maintain the comparability. In our study, we used serum collected and processed similarly in all subjects; thus, the difference between ACS patients and their healthy controls were analysed and used in statistical analysis [4].

Every sample collection and processing methods have its limitations and challenges; plasma is processed from blood by using anticoagulants, and especially EDTA processing interferes and decreases MMP activity [1]. Different blood fluids may describe the inflammatory and disease status slightly

Associate Editor Enrique Lara-Pezzi oversaw the review of this article

Laura Lahdentausta

laura.lahdentausta@helsinki.fi

1 Department of Oral and Maxillofacial Diseases, University of Helsinki and Helsinki University Hospital, Biomedicum Helsinki 1, Haartmaninkatu 8, 00290 Helsinki, Finland

2 University of Helsinki, PL 63, 00014 Helsinki, Finland

3 Division of Periodontology, Department of Dental Medicine, Karolinska Institutet, Huddinge, Sweden

4 Skåne University, Lund, Skåne, Sweden

5 Department of Paediatrics, Division of Paediatric Cardiology, Skåne University Hospital, Lund, Sweden differently; serum concentrations include the free circulating and "secretable" MMP-9 and TIMP-1, whereas EDTA plasma contains the EDTA-inhibited concentrations. The MMP-9 and TIMP-1 concentrations of cases and controls should be measured from same sample material collected and processed similarly through study.

Compared to plasma, MMP-9 and TIMP-1 are released to serum due to complement, contact, and platelet activation during the coagulation [1]. These processes are important in haemostasis and thrombosis, and they are also involved in inflammation and innate immune response. The elevated MMP-9 concentrations in serum compared to plasma may describe the inflammatory burden or "reservoir", i.e. the inflammatory potential. Therefore, serum MMP-9 and TIMP-1 have great potential to become cardiac biomarkers in clinical use.

Funding This study was funded by grants from the Finnish Dental Society Apollonia (LL and PJP), Orion Research Foundation (LL), Juhani Aho Medical Foundation (LL), Aarne and Aili Turunen Foundation (LL), the Association of Finnish Female Dentists (LL), the Academy of Finland (\#1266053) (PJP), the Sigrid Juselius foundation (PJP), the Aarne Koskelo foundation (PJP), the Päivikki and Sakari Sohlberg foundation (PJP), the Lund University (EP), the Lund University Hospital (EP), the Helsinki University Hospital Research Foundation (TYH 2016251, 2017251, 2018229, Y1149SUL32), and the Karolinska Institutet, Stockholm, Sweden.

\section{Compliance with Ethical Standards}

Conflict of Interest The authors declare that they have no conflict of interest.

Ethical Approval This article does not contain any studies with human participants or animals performed by any of the authors.

\section{References}

1. Alby, C., Ben Abdesselam, O., Foglietti, M. J., \& Beaudeux, J. L. (2002). Preanalytical aspects regarding the measurement of metalloproteinase- 9 and tissue inhibitor or metalloproteinase-1 in blood. Clinica Chimica Acta, 325(1-2), 183-186. 
2. Gerlach, R. F., Uzuelli, J. A., Souza-Tarla, C. D., \& Tanus-Santos, J. E. (2005). Effect of anticoagulants on the determination of plasma matrix metalloproteinase (MMP)-2 and MMP-9 activities. Analytical Biochemistry, 344(1), 147-149.

3. Jung, K., Laube, C., Lein, M., Lichtinghagen, R., Tschesche, H., Schnorr, D., \& Loening, S. A. (1998). Kind of sample as preanalytical determinant of matrix metalloproteinase 2 and 9 and tissue inhibitor of metalloproteinase 2 in blood. Clinical Chemistry, 44(5), 1060-1062.
4. Lahdentausta, L., Leskela, J., Winkelmann, A., Tervahartiala, T., Sorsa, T., Pesonen, E., \& Pussinen, P. J. (2018). Serum MMP-9 diagnostics, prognostics, and activation in acute coronary syndrome and its recurrence. Journal of Cardiovascular Translational Research, 11(3), 210-220. https://doi.org/10.1007/s12265-0189789-x.

5. Santana, I., \& Tanus-Santos, J. (2018). Serum or plasma matrix metalloproteinase (MMP)-9 levels and cardiovascular diseases. Journal of Cardiovascular Translational Research. 